\title{
Optimizing Stroke and Bleeding Risk Assessment in Patients with Atrial Fibrillation: A Balance of Evidence, Practicality and Precision
}

\author{
Marco Proietti ${ }^{1,2}$ Nebojsa Mujovic ${ }^{3,4}$ Tatjana S. Potpara ${ }^{3,4}$ \\ ${ }^{1}$ Istituto di Ricerche Farmacologiche "Mario Negri” IRCCS, Milan, Italy \\ 2 Department of Internal Medicine and Medical Specialties, Sapienza \\ University of Rome, Rome, Italy \\ ${ }^{3}$ School of Medicine, University of Belgrade, Belgrade, Serbia \\ ${ }^{4}$ Cardiology Clinic, Clinical Centre of Serbia, University of Belgrade, \\ Belgrade, Serbia
}

Thromb Haemost 2018;118:2014-2017.

Optimal stroke prevention using oral anticoagulant (OAC) therapy is essential for the management of patients with atrial fibrillation (AF) who are at increased risk of stroke, but decision making on thromboprophylaxis requires evaluation of individual patient's thromboembolic and bleeding risks. ${ }^{1}$ Several risk assessment tools have been developed to streamline the evaluation of patients with AF but navigating through the labyrinth of options may be confusing for clinicians. ${ }^{2}$

\section{Stroke Risk in AF: Evidence versus Practical Decision Making}

In this issue of Thrombosis Haemostasis, Borre et $\mathrm{al}^{3}$ reported a comprehensive systematic review and meta-analysis commissioned by the Patient-Centred Outcomes Research Institute to update a 2013 Agency for Healthcare Research and Quality review ${ }^{4}$ of the comparative accuracy and impact on clinical decision making of several thromboembolic and bleeding risk assessment tools in patients with AF.

They addressed two key topics: (1) the comparative diagnostic accuracy and impact on clinical decision making of available clinical and imaging tools and associated risk factors for predicting thromboembolic risk; and (2) the comparative diagnostic accuracy and impact on clinical decision making of clinical tools and associated risk factors for predicting bleeding events. The authors conducted an extensive literature search (from 2000 to 2018) and evidence appraisal of published data for clinical stroke scores $\left(\mathrm{CHADS}_{2}, \mathrm{CHA}_{2} \mathrm{DS}_{2}-\mathrm{VASc}\right.$, Framingham and age, biomarkers and clinical history $[\mathrm{ABC}]$-stroke), cardiac imaging predictors and clinical bleeding risk scores (HAS-BLED, HEMOR$\mathrm{R}_{2}$ HAGES, ATRIA, Bleeding Risk Index and ABC-bleeding).

received

October 31, 2018

accepted

October 31, 2018

Address for correspondence Tatjana S. Potpara, MD, PhD, Cardiology Clinic, Clinical Centre of Serbia, Visegradska 26, 11000 Belgrade, Serbia (e-mail: tatjana.potpara@med.bg.ac.rs).

Not surprisingly, the CHADS 2 and $\mathrm{CHA}_{2} \mathrm{DS}_{2}$-VASc scores were the most commonly evaluated stroke risk assessment tools (29 and 24 studies, respectively). Overall, the $\mathrm{CHADS}_{2}$, $\mathrm{CHA}_{2} \mathrm{DS}_{2}$-VASc and ABC-stroke scores had the best evidence for prediction ability for stroke-however, notwithstanding the heterogeneity among studies included in the metaanalyses, the $\mathrm{CHADS}_{2}, \mathrm{CHA}_{2} \mathrm{DS}_{2}$-VASc, Framingham and ABC-stroke scores all had a modest prediction ability for thromboembolic events, with c-statistic values ranging from 0.63 (Framingham) to 0.69 (continuous $\mathrm{CHADS}_{2}$ score). ${ }^{3}$ This is in line with previous meta-analyses ${ }^{5-8}$ showing broadly similar performance of various clinical risk factor-based stroke scores (including the $\mathrm{CHA}_{2} \mathrm{DS}_{2}$-VASc) in prediction of thromboembolic events. However, the difference of the $\mathrm{CHA}_{2} \mathrm{DS}_{2}$-VASc in comparison to other stroke scores is its relative accuracy in identifying $\mathrm{AF}$ patients at truly low risk of stroke (i.e. $\mathrm{CHA}_{2} \mathrm{DS}_{2}$-VASc of 0 in men, or 1 in women) who do not need any anti-thrombotic therapy. ${ }^{8-10}$

Recognizing that all clinical scores only have modest predictive value for high-risk patients that sustain events and that current risk scores are designed to be simple and reductionist, the use of a simplified, clinical risk factor-based approach to the management of AF-related stroke risk has been acknowledged by recent international AF guidelines. ${ }^{1,11}$ The default should be to 'offer stroke prevention, unless the patient is "low risk". A simple message is needed, as guideline-adherent treatment has been associated with improved outcomes in multiple AF cohorts. ${ }^{12-16}$

The presence of even a single $\mathrm{CHA}_{2} \mathrm{DS}_{2}$-VASc risk factor is associated with an excess in stroke and mortality, ${ }^{17,18}$ and OAC use is associated with positive net clinical benefit in comparison to aspirin (which is harmful) or no therapy. ${ }^{19}$ Variations in

(c) 2018 Georg Thieme Verlag KG Stuttgart · New York
DOI https://doi.org/ 10.1055/s-0038-1676074. ISSN 0340-6245. 
reported event rates for patients' single-risk-factor AF cohorts have caused debate ${ }^{20}$ but may reflect different study settings (hospital vs. community), not all risk factors carrying equal risk, methodological errors (with some papers 'conditioning on the future' by excluding patients who had ever started OAC even during follow-up) and the inclusion of females (who have 1 point, yet low risk) in reports of event rates with $\mathrm{CHA}_{2} \mathrm{DS}_{2}$-VASc score of $1 .{ }^{16,21}$ Indeed, female sex is rather a risk modifier than an independent stroke risk factor and becomes relevant only in the presence of other $\mathrm{CHA}_{2} \mathrm{DS}_{2}$-VASc factors ${ }^{22}$; however, ignoring female sex (i.e. using the $\mathrm{CHA}_{2} \mathrm{DS}_{2}$-VA score, without ' $\mathrm{Sc}^{, 23}$ ) could under-estimate stroke risk in females with $\mathrm{AF}^{24}$

As mentioned, clinical risk factor-based scores generally have a modest prediction ability for the outcome event of interest. Adding various biomarkers ('biological markers') to clinical scores-that is, blood biomarkers (e.g. brain natriuretic peptide, cardiac troponin, creatinine, etc.), cardiac or cerebral imaging (echocardiographic, magnetic resonance, etc.) or electrocardiographic indices in sinus rhythm (e.g., the $P$ wave axis ${ }^{25}$ )-improve the score's prediction ability, at least statistically, but such complexity may be impractical or unfeasible outside a highly structured setting. ${ }^{2}$

\section{Vascular Disease and Stroke Risk}

Optimal acknowledgment of clinical information pertaining to specific $\mathrm{CHA}_{2} \mathrm{DS}_{2}$-VASc score component is crucial for proper stroke risk assessment in each patient. For example, the 'V' (vascular disease) traditionally includes validated factors such as previous myocardial infarction, complex aortic plaque or peripheral artery disease.

In this same issue of Thrombosis Haemostasis, Steensig et $\mathrm{al}^{26}$ extend their recent report on significant association between the presence of angiographically documented coronary artery disease (CAD) and subsequent thromboembolic events ${ }^{27}$ showing that the extent of CAD (i.e. 1-, 2-, 3-vessel or diffuse) did not add additional risk prediction information regarding ischaemic stroke, transient ischaemic attack or systemic embolism among 12,690 AF patients undergoing coronary angiography (with CAD diagnosed in 59.4\%) over a 3-year follow-up. Hence, angiographically documented CAD should be included in the ' $\mathrm{V}$ ' component of the $\mathrm{CHA}_{2} \mathrm{DS}_{2}$ VASc score. This would translate to an indication for OAC use in $3 \%$ of low-risk patients according to the European AF guidelines, or in $6 \%$ per the U.S. guidelines. Non-invasively diagnosed CAD using contrast angiography or stress testing would probably have the same significance, albeit not investigated in the present study.

\section{Bleeding Risk Assessment}

Bleeding risk assessment is a sensitive part of risk evaluation in AF patients that is sometimes misinterpreted or even abused. ${ }^{28}$ All international AF guidelines recommend bleeding risk assessment but beyond listing the bleeding risk factors, practical guidance on clinical decision making on

\section{The Atrial fibrillation Better Care (ABC) pathway for integrated care management}

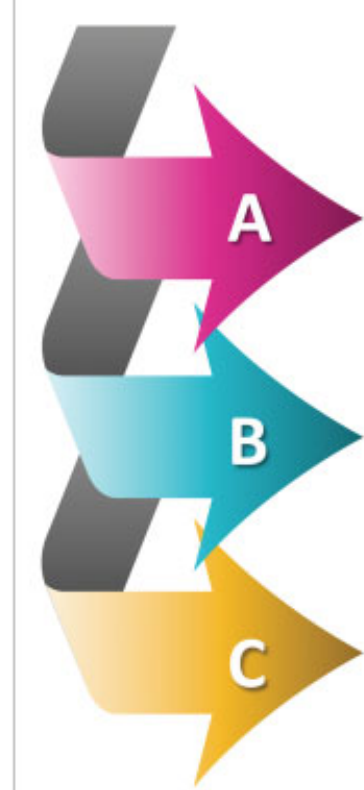

Avoid stroke

Optimize stroke prevention ("Birmingham 3-step").

Better symptom management Treat symptoms.

\section{Cardiovascular} and other comorbidities/risk factors

Manage risk factors and comorbidities.
Step 1: Identify low-risk patients.

Step 2: Offer stroke prevention to patients with $\geq 1$ risk factors for stroke. Assess bleeding risk.

Step 3: Decide on OAC (either a VKA with wellmanaged TTR or a NOAC).

Patient-centered and symptom-directed decisions on rate versus rhythm control.

Manage hypertension, heart failure, diabetes mellitus, cardiac ischaemia and sleep apnea.

Lifestyle changes: obesity reduction, regular exercise and reduction of alcohol/stimulant use.

Patient psychological morbidity.

Consider patient values and preferences.

Fig. 1 The Atrial fibrillation Better Care (ABC) pathway. NOAC, non-vitamin K antagonist oral anticoagulant; OAC, oral anticoagulant; TTR, time in therapeutic range; VKA, vitamin $\mathrm{K}$ antagonist. 
OAC use in high-risk AF patients at increased risk of bleeding is less well specified. ${ }^{1}$

In their systematic review and evidence appraisal, Borre et $\mathrm{al}^{3}$ also explored 38 studies that reported on bleeding risk in AF patients. Overall, they found moderate strength of evidence on increased bleeding risk in AF patients with chronic kidney disease, and that the HAS-BLED score provided the best prediction of bleeding events among the investigated bleeding risk assessment tools. Indeed, the HAS-BLED score has been extensively validated in various setting, including AF patients on OAC (either vitamin $\mathrm{K}$ antagonists [VKA] or non-VKA OACs), aspirin or no antithrombotic therapy, with respect to various bleeding outcomes (i.e. major bleeding or intracranial haemorrhage). ${ }^{28}$

Bleeding risk assessment using the HAS-BLED score is not meant to preclude the use of OAC, but to identify high-risk patients in whom modifiable bleeding risk factors such as uncontrolled hypertension $(\mathrm{H})$, labile international normalized ratios in patient taking VKAs (L), concomitant use of non-steroidal anti-inflammatory drugs, anti-platelet drugs or excessive alcohol consumption (D) can be addressed or modified, and who need clinical follow-up earlier rather than later (e.g. 4 weeks rather than 4-6 months). Importantly, using a formal bleeding risk assessment tool (i.e. the HASBLED score) had significantly better predictive value for bleeding risk assessment compared with the approach based only on modifiable bleeding risk factors. ${ }^{29-31}$

\section{The Dynamic Nature of Stroke and Bleeding Risk}

Many studies of risk factors and biomarkers have investigated something at study entry ('baseline') and ascertained outcomes many years later (sometimes 5 or 10 years). The patient's clinical risk profile changes over time and this change has been shown to have better prediction ability for the respective risk than simply relying on the baseline score values. ${ }^{32,33}$ Hence, neither thromboembolic nor bleeding risks are static and must be re-assessed regularly. This is part of a comprehensive and holistic approach to the management of patients with $\mathrm{AF}^{34}$ (see - Fig. 1).

\section{Conflict of Interest}

None.

\section{References}

1 Lip G, Freedman B, De Caterina R, Potpara TS. Stroke prevention in atrial fibrillation: Past, present and future. Comparing the guidelines and practical decision-making. Thromb Haemost 2017;117 (07):1230-1239

2 Lip GY. Stroke and bleeding risk assessment in atrial fibrillation: when, how, and why? Eur Heart J 2013;34(14):1041-1049

3 Borre ED, Goode A, Raitz G, et al. Predicting thromboembolic and bleeding event risk in patients with non-valvular atrial fibrillation: a systematic review. Thromb Haemost 2018;118(12). Doi: $10.1055 / \mathrm{s}-0038-1675400$

4 Lopes RD, Crowley MJ, Shah BR, et al. Stroke Prevention in Atrial Fibrillation. Rockville, MD: Agency for Healthcare Research and
Quality (US); 2013. Report No.: 13-EHC113-EF. AHRQ Comparative Effectiveness Reviews.

5 Chen JY, Zhang AD, Lu HY, Guo J, Wang FF, Li ZC. CHADS2 versus CHA2DS2-VASc score in assessing the stroke and thromboembolism risk stratification in patients with atrial fibrillation: a systematic review and meta-analysis. J Geriatr Cardiol 2013;10 (03):258-266

6 Xiong Q, Chen S, Senoo K, Proietti M, Hong K, Lip GY. The CHADS2 and CHA2DS2-VASc scores for predicting ischemic stroke among East Asian patients with atrial fibrillation: a systemic review and meta-analysis. Int J Cardiol 2015;195: 237-242

7 Zhu WG, Xiong QM, Hong K. Meta-analysis of CHADS2 versus CHA2DS2-VASc for predicting stroke and thromboembolism in atrial fibrillation patients independent of anticoagulation. Tex Heart Inst J 2015;42(01):6-15

8 Zhu W, Fu L, Ding Y, et al. Meta-analysis of ATRIA versus $\mathrm{CHA}_{2} \mathrm{DS}_{2}-$ VASc for predicting stroke and thromboembolism in patients with atrial fibrillation. Int J Cardiol 2017;227:436-442

9 Olesen JB, Torp-Pedersen C, Hansen ML, Lip GY. The value of the CHA2DS2-VASc score for refining stroke risk stratification in patients with atrial fibrillation with a CHADS2 score 0-1: a nationwide cohort study. Thromb Haemost 2012;107(06): 1172-1179

10 Potpara TS, Polovina MM, Licina MM, Marinkovic JM, Prostran MS, Lip GY. Reliable identification of "truly low" thromboembolic risk in patients initially diagnosed with "lone" atrial fibrillation: the Belgrade atrial fibrillation study. Circ Arrhythm Electrophysiol 2012;5(02):319-326

11 Lip GYH, Banerjee A, Boriani G, et al. Antithrombotic therapy for atrial fibrillation: CHEST Guideline and Expert Panel Report. Chest 2018:S0012-3692(18)32244-X

12 Lip GY, Laroche C, Popescu MI, et al. Improved outcomes with European Society of Cardiology guideline-adherent antithrombotic treatment in high-risk patients with atrial fibrillation: a report from the EORP-AF General Pilot Registry. Europace 2015; 17:1777-1786

13 Proietti M, Nobili A, Raparelli V, Napoleone L, Mannucci PM, Lip GY; REPOSI investigators. Adherence to antithrombotic therapy guidelines improves mortality among elderly patients with atrial fibrillation: insights from the REPOSI study. Clin Res Cardiol 2016; 105(11):912-920

$14 \mathrm{Li} \mathrm{CH}$, Liu CJ, Chou AY, et al. European Society of Cardiology guideline-adherent antithrombotic treatment and risk of mortality in Asian patients with atrial fibrillation. Sci Rep 2016; 6:30734

15 Mazurek M, Shantsila E, Lane DA, Wolff A, Proietti M, Lip GYH. Guideline-adherent antithrombotic treatment improves outcomes in patients with atrial fibrillation: insights from the Community-Based Darlington Atrial Fibrillation Registry. Mayo Clin Proc 2017;92(08):1203-1213

16 Nielsen PB, Larsen TB, Skjøth F, Overvad TF, Lip GY. Stroke and thromboembolic event rates in atrial fibrillation according to different guideline treatment thresholds: a nationwide cohort study. Sci Rep 2016;6:27410

17 Olesen JB, Torp-Pedersen C. Stroke risk in atrial fibrillation: do we anticoagulate CHADS2 or CHA2DS2-VASc $\geq 1$, or higher? Thromb Haemost 2015;113(06):1165-1169

18 Fauchier L, Lecoq C, Clementy N, et al. Oral anticoagulation and the risk of stroke or death in patients with atrial fibrillation and one additional stroke risk factor: the Loire Valley Atrial Fibrillation Project. Chest 2016;149(04):960-968

19 Lip GY, Skjøth F, Nielsen PB, Larsen TB. Non-valvular atrial fibrillation patients with none or one additional risk factor of the CHA2DS2-VASc score. A comprehensive net clinical benefit analysis for warfarin, aspirin, or no therapy. Thromb Haemost 2015;114(04):826-834 
20 Potpara TS, Dagres N, Mujović N, et al. Decision-making in clinical practice: oral anticoagulant therapy in patients with non-valvular atrial fibrillation and a single additional stroke risk factor. Adv Ther 2017;34(02):357-377

21 Nielsen PB, Lip GY. Adding rigor to stroke rate investigations in patients with atrial fibrillation. Circulation 2017;135(03): 220-223

22 Nielsen PB, Skjøth F, Overvad TF, Larsen TB, Lip GYH. Female sex is a risk modifier rather than a risk factor for stroke in atrial fibrillation: should we use a $\mathrm{CHA}_{2} \mathrm{DS}_{2}$-VA score rather than $\mathrm{CHA}_{2} \mathrm{DS}_{2}$-VASc? Circulation 2018;137(08):832-840

23 Brieger D, Amerena J, Attia J, et al; NHFA CSANZ Atrial Fibrillation Guideline Working Group. National Heart Foundation of Australia and the Cardiac Society of Australia and New Zealand: Australian Clinical Guidelines for the Diagnosis and Management of Atrial Fibrillation 2018. Heart Lung Circ 2018;27(10):1209-1266

24 Overvad TF, Potpara TS, Nielsen PB. Stroke risk stratification: $\mathrm{CHA}_{2} \mathrm{DS}_{2}$-VA or $\mathrm{CHA}_{2} \mathrm{DS}_{2}$-VASc? Heart Lung Circ 2018:S14439506(18)31866-3

25 Maheshwari A, Norby FL, Roetker NS, et al. Refining prediction of atrial fibrillation-related stroke using the $\mathrm{P}_{2}-\mathrm{CHA}_{2} \mathrm{DS}_{2}-\mathrm{VASC}$ score: The atherosclerosis risk in communities (ARIC) study and multi-ethnic study of atherosclerosis (MESA). Circulation 2018. Doi: 10.1161/CIRCULATIONAHA.118.035411

26 Steensig K, Olesen KKW, Thim T, et al. Should the presence or extent of coronary artery disease be quantified in the $\mathrm{CHA}_{2} \mathrm{DS}_{2}$ VASc score in atrial fibrillation? A report from the western Denmark heart registry. Thromb Haemost 2018. Doi: 10.1055/ s-0038-1675401
27 Steensig K, Olesen KKW, Thim T, et al. Coronary artery disease is independent risk factor for stroke among patients with atrial fibrillation. J Am Coll Cardiol 2018:S0735-1097(18)36961-4

28 Lip GY, Lane DA. Bleeding risk assessment in atrial fibrillation: observations on the use and misuse of bleeding risk scores. J Thromb Haemost 2016;14(09):1711-1714

29 Esteve-Pastor MA, Rivera-Caravaca JM, Shantsila A, Roldán V, Lip GYH, Marín F. Assessing bleeding risk in atrial fibrillation patients: comparing a bleeding risk score based only on modifiable bleeding risk factors against the HAS-BLED score. The AMADEUS Trial. Thromb Haemost 2017;117(12):2261-2266

30 Guo Y, Zhu H, Chen Y, Lip GYH. Comparing bleeding risk assessment focused on modifiable risk factors only versus validated bleeding risk scores in atrial fibrillation. Am J Med 2018;131(02):185-192

31 Chao TF, Lip GYH, Lin SL, et al. Major bleeding and intracranial haemorrhage risk prediction in patients with atrial fibrillation: attention to modifiable bleeding risk factors or use of a bleeding risk stratification score? A nationwide cohort study. Int J Cardiol 2018;254:157-161

32 Chao TF, Lip GYH, Liu CJ, et al. Relationship of aging and incident comorbidities to stroke risk in patients with atrial fibrillation. J Am Coll Cardiol 2018;71(02):122-132

33 Chao TF, Lip GYH, Lin YJ, et al. Incident risk factors and major bleeding in patients with atrial fibrillation treated with oral anticoagulants: a comparison of baseline, follow-up and delta HAS-BLED scores with an approach focused on modifiable bleeding risk factors. Thromb Haemost 2018;118(04):768-777

34 Lip GYH. The ABC pathway: an integrated approach to improve AF management. Nat Rev Cardiol 2017;14(11):627-628 